\title{
Design of an ozone and nitrogen dioxide sensor unit and its long-term operation within a sensor network in the city of Zurich - supplementary materials
}

\author{
Mueller Michael $^{1}$, Meyer Jonas ${ }^{2}$, and Hueglin Christoph ${ }^{1}$ \\ ${ }^{1}$ Empa, Swiss Federal Institute for Materials Science and Technology, Duebendorf, Switzerland. \\ ${ }^{2}$ Decentlab GmbH, Duebendorf, Switzerland. \\ Correspondence to: M. Mueller (michael.mueller@empa.ch)
}

1 Network of sensor units

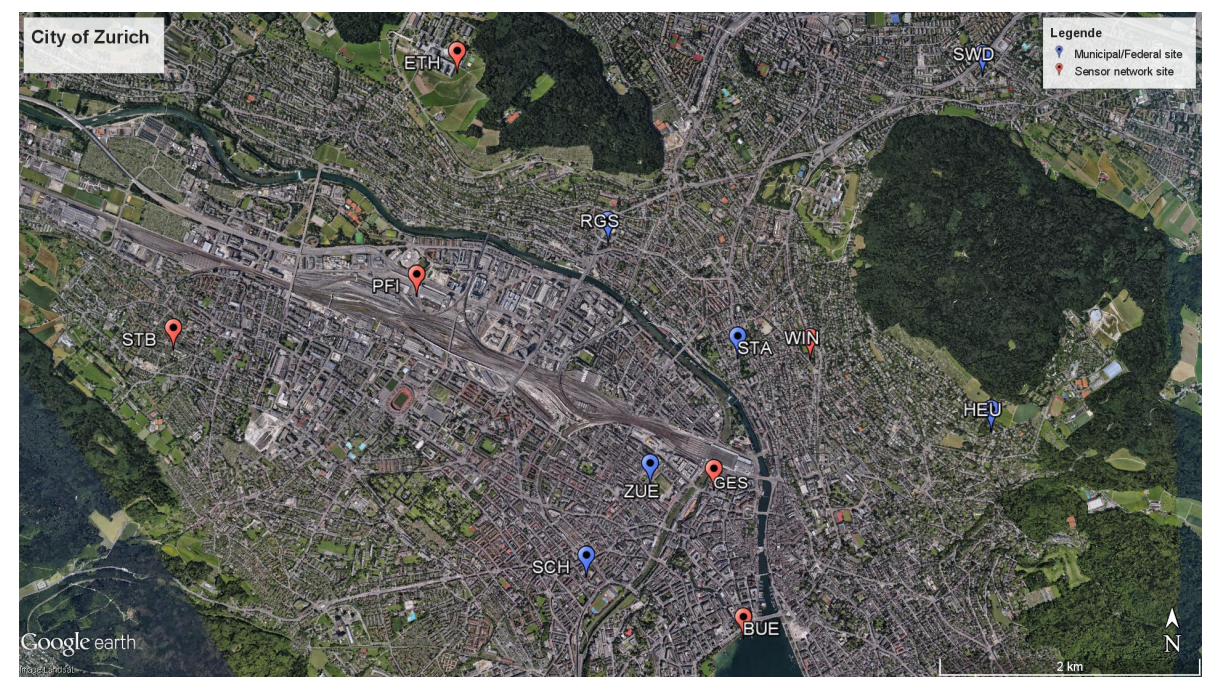

Figure 1. Map depicting the locations of the sensor units within the sensor network (red symbols) as well as of the municipal and federal air quality monitoring stations (blue symbols). The map was created with Google Earth. 

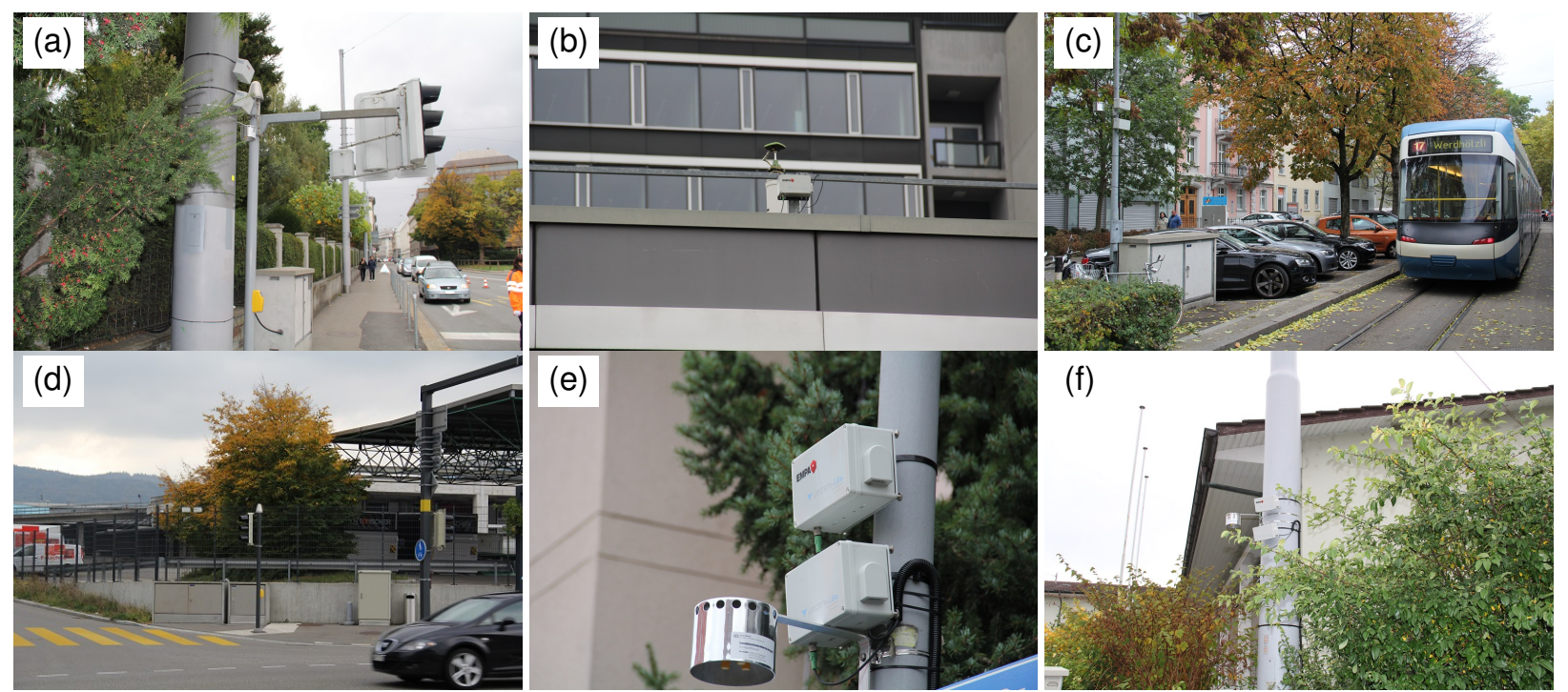

Figure 2. Pictures of the six locations where sensor units were operated: (a) BUE, (b) ETH, (c) GES, (d) PFI, (e) STB, (f) WIN. The pictures show the sensor units as well as the mounting of the $\mathrm{NO}_{2}$ passive samplers. 
(a)

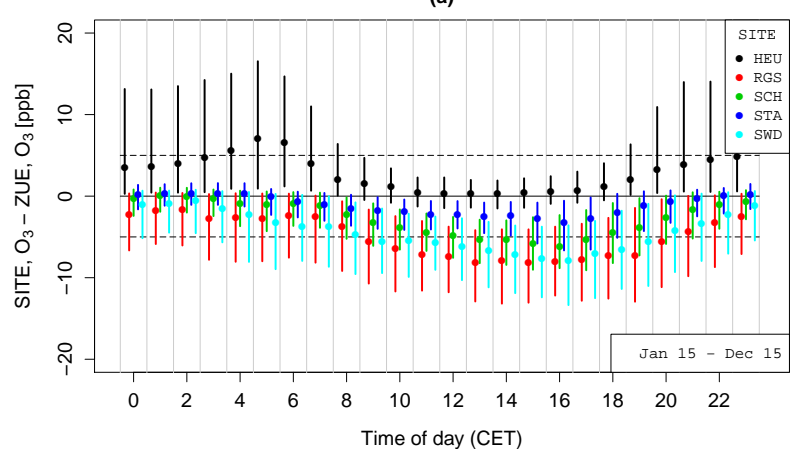

(b)

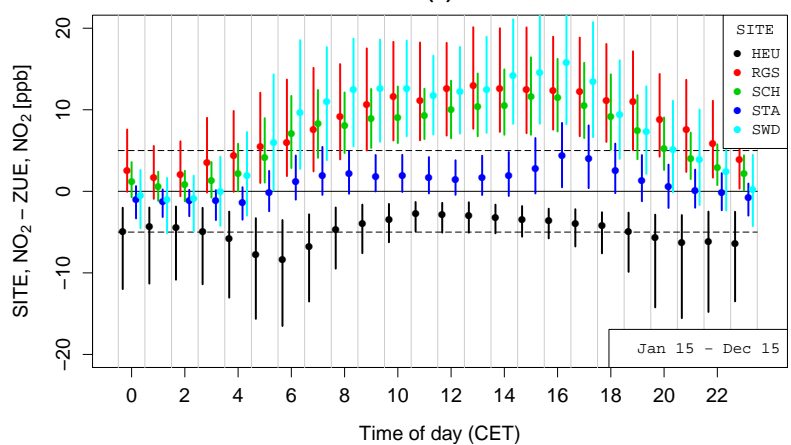

(c)

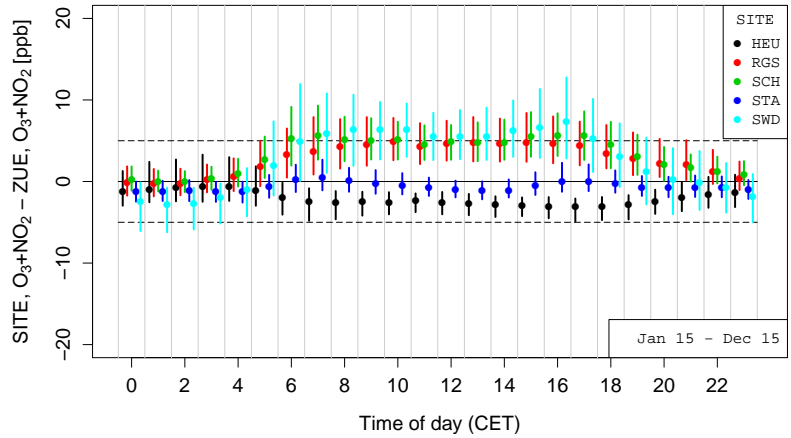

Figure 3. Differences in 30 minutes values of $\mathrm{O}_{3}(\mathrm{a}), \mathrm{NO}_{2}$ (b) and the sum of $\mathrm{NO}_{2}$ and $\mathrm{O}_{3}$ (c) concentration between sites $\mathrm{HEU}$, RGS, $\mathrm{SCH}$, STA and SWD and site ZUE, respectively, in 2015. The points depict the respective median difference and the lines depict the range between the $25 \%$ and the $75 \%$ quantiles of the differences. 
(a)

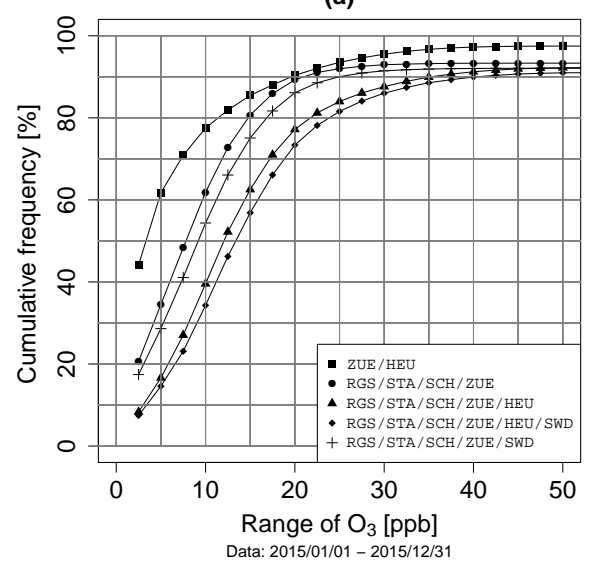

(b)

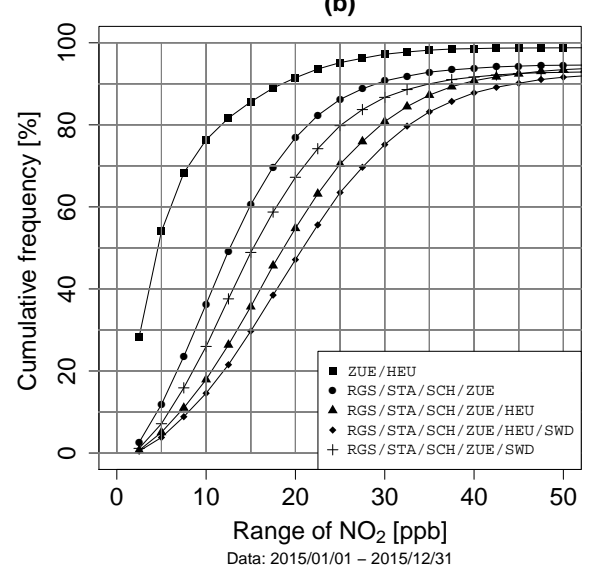

Figure 4. Cumulative distribution of $\mathrm{O}_{3}$ (a) and $\mathrm{NO}_{2}$ (b) concentration ranges. Range of $\mathrm{NO}_{2}$ and $\mathrm{O}_{3}$ denote the difference between the maximum and the minimum average 30 minutes $\mathrm{NO}_{2}$ and $\mathrm{O}_{3}$ concentrations, respectively, measured at AQM sites being part of five defined site groups. The sites being part of each group are listed in the legend. $100 \%$ refers to the maximum possible number of 30 minutes values in the year 2015. Cumulative distributions remain below $100 \%$ due to calibration and maintenance periods and instrument failures. 


\section{Sensor calibration}
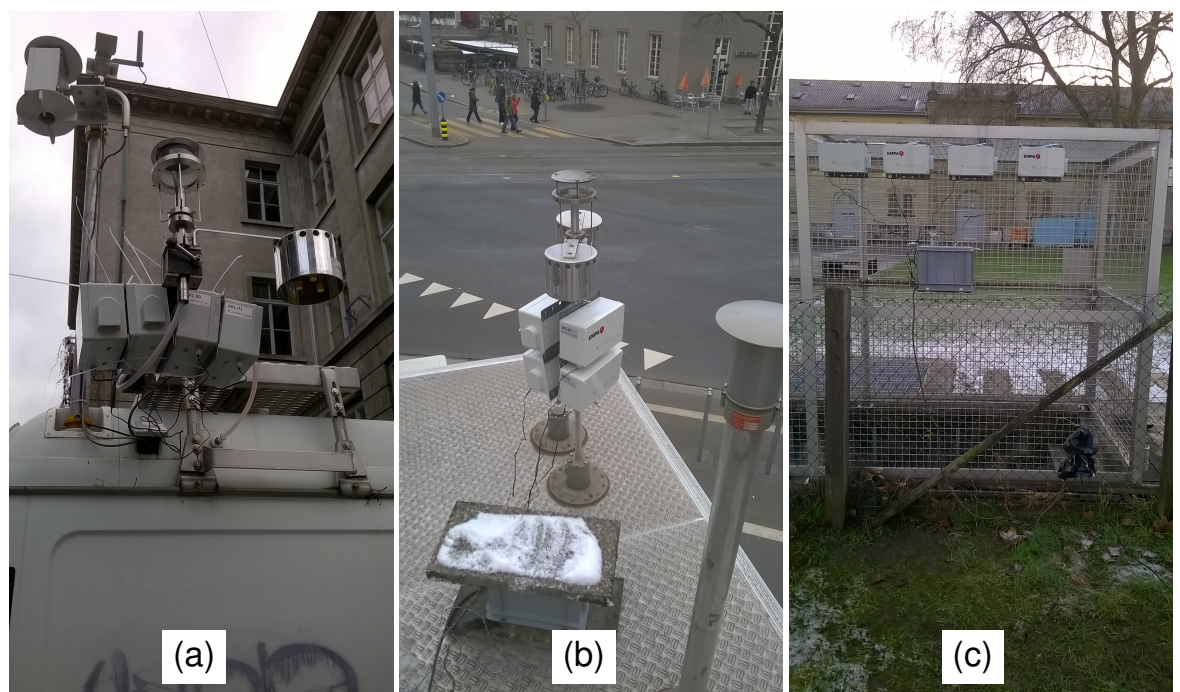

Figure 5. Pictures of the sensor units running in parallel with instruments of the air quality monitoring stations RGS (a), SCH (b) and ZUE (c) during sensor calibration. 


\section{Processing of the data from the sensor units}
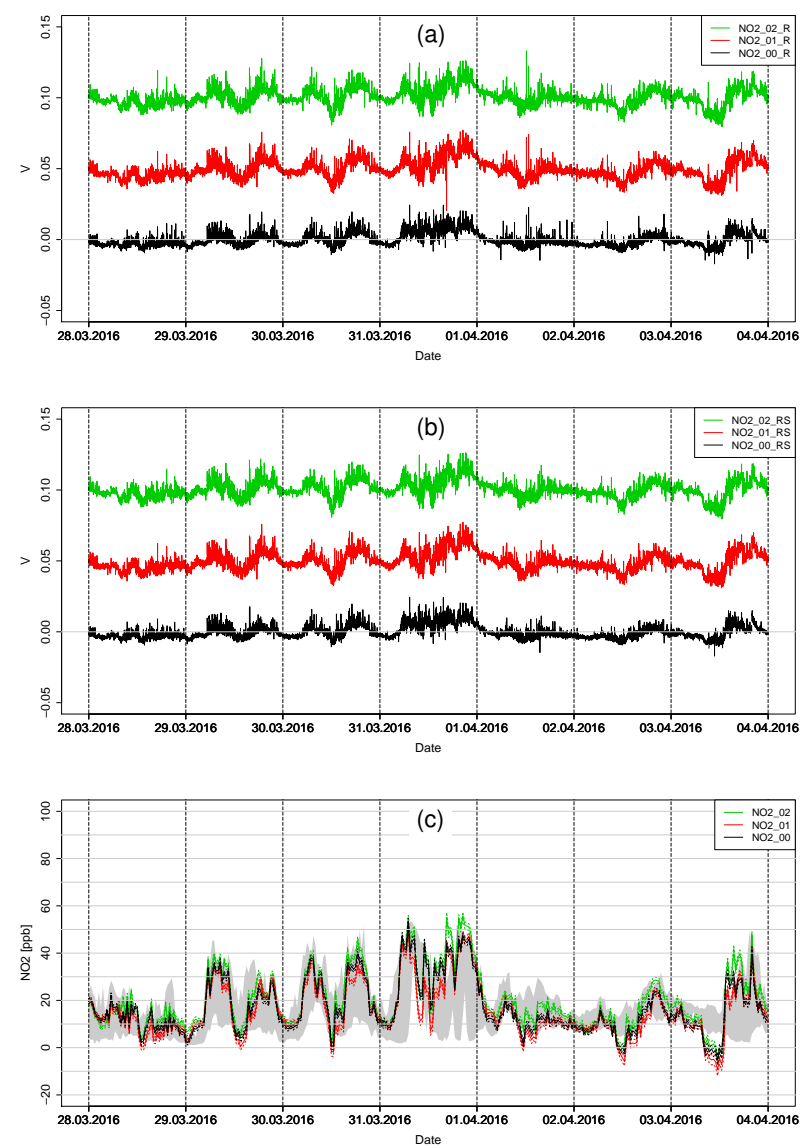

Figure 6. Illustrative example of the data processing of $\mathrm{NO}_{2}$ sensors from the sensor units. (a) Raw sensor data of the three $\mathrm{NO}_{2}$ sensors integrated in the sensor units back-transformed to the Volt scale (1 minute values). Time-series are shifted by $50 \mathrm{mV}$, respectively, in order to improve the clarity. (b) Pre-processed sensor output (1 minute values). (c) Final $\mathrm{NO}_{2}$ time-series of 30 minutes mean values. The band depicted in grey indicates the range of the 30 minutes mean $\mathrm{NO}_{2}$ concentrations measured at the AQM sites HEU, RGS, SCH, STA and ZUE (see Fig. 1 for the respective site locations). 
(a)
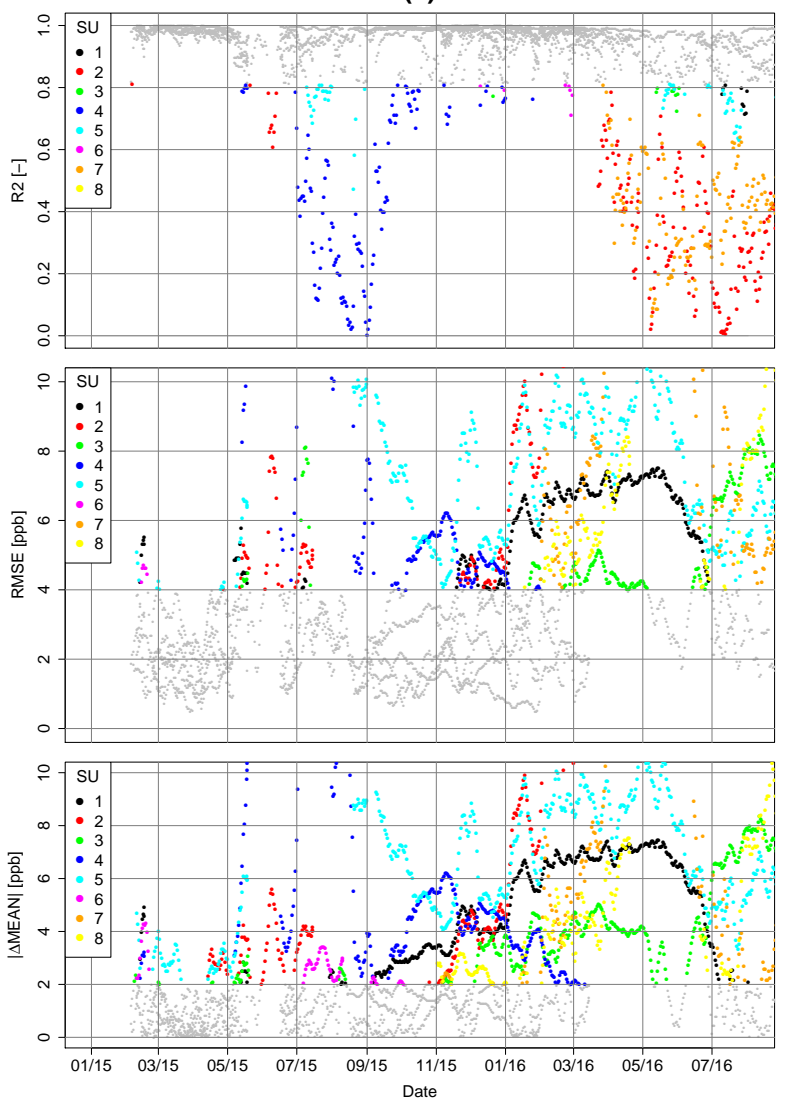

(b)
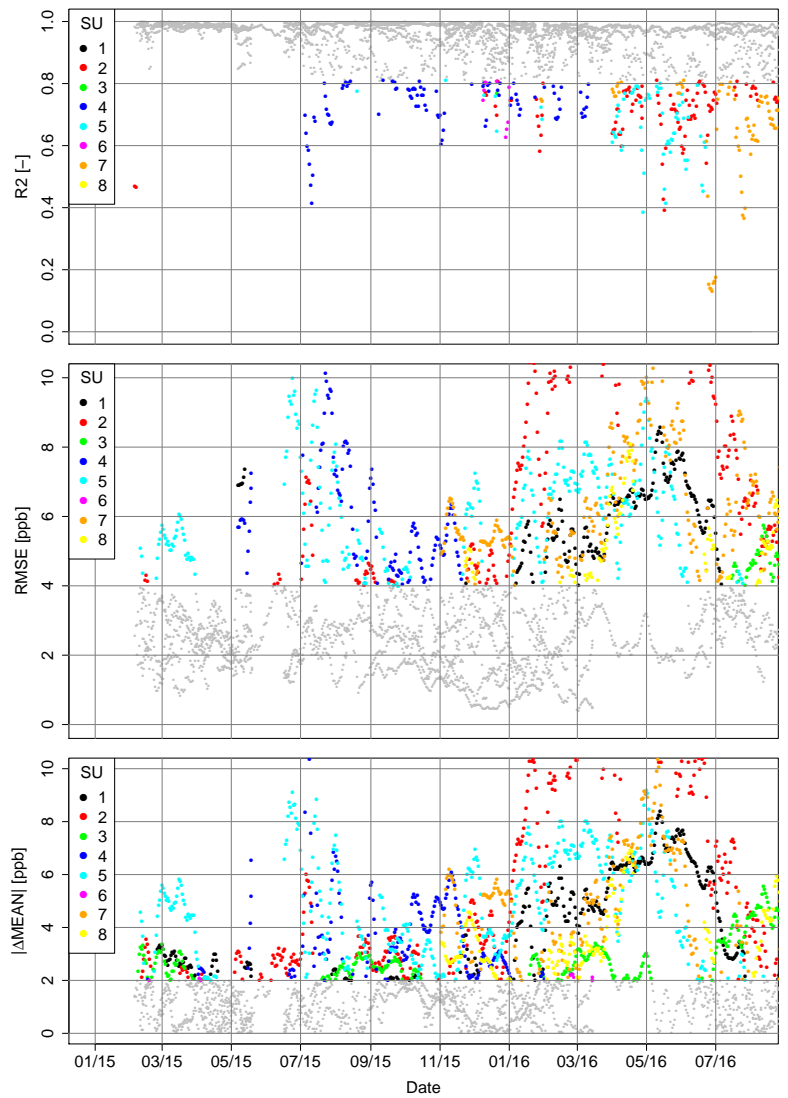

Figure 7. Comparison of measurements from the redundant $\mathrm{O}_{3}$ sensors integrated in the SUs by means of the 7 days rolling R2, root mean square and absolute average difference based on 30 minutes $\mathrm{NO}_{2}$ concentrations. There are $n(n-1) / 2$ values for $n$ integrated sensors per day. Only time periods are considered when the SUs were calibrated or operated in the sensor net. The values in (a) are derived from data set 1 which is based on a linear fit only. The values in (b) are derived from data set 3 (initial and remote calibration based on Eq. 6). 